\title{
Random Fuzzy Decision Models for Pharmaceutical R\&D Project Investment under Uncertainty
}

\author{
Changsheng $\mathrm{Yi}^{1} \&$ Qiumei $\mathrm{Jin}^{2}$ \\ ${ }^{1}$ School of Management Science and Engineering, Anhui University of Technology, Ma'anshan, China \\ ${ }^{2}$ The Hospital of Anhui University of Technology, Ma'anshan, China \\ Correspondence: Changsheng Yi, School of Management Science and Engineering, Anhui University of Technol- \\ ogy, Ma'anshan, China. E-mail: yics2005@163.com
}

\author{
Received: December 31, 2014 Accepted: January 21, 2015 Online Published: January 29, 2015 \\ doi:10.5539/ijsp.v4n1p170 URL: http://dx.doi.org/10.5539/ijsp.v4n1p170
}

The research is financed by a grant from the Humanities and Social Sciences Foundation of the Minis-try of Education (10YJC630352), a grant from the Humanities and Social Sciences Major Program of Anhui Province (SK2014ZD018), and a grant from the Jinglong Company Research Project (RD13210009).

\begin{abstract}
This paper considers the models of optimal stopping time for pharmaceutical R\&D investment without rivalry in random fuzzy environments. Specifically, the R\&D process can be described as a jump diffusion process of scientific knowledge. Jump events can be characterized by the technological breakthroughs or innovative discovery. In previous $R \& D$ literature, the time intervals until next jumps are generally assumed as random variables observed exponentially distributed. Here, the inter-arrival times are regarded as random fuzzy variables observing arbitrary distributions. Moreover, the decision variable is the stopping time which determines when the project should be terminated. Three frameworks of project return performance (expected net return, $\alpha$-optimistic net return and return reliability) are proposed and three kinds of random fuzzy programming models are established to model the different R\&D investment decision problems according to the decision-maker's attitude. Considering the complexity of these models, the random fuzzy simulation is employed to obtain the values of project return performance and the simultaneous perturbation stochastic approximation (SPSA) algorithm is designed to solve the optimal stopping problems. Finally, the effectiveness of the hybrid algorithm and the applicability of these models are illustrated by some numerical examples.
\end{abstract}

Keywords: pharmaceutical R\&D project, optimal stopping, random fuzzy variable, hybrid intelligent algorithm

\section{Introduction}

With the increasing competitive and fast-growing marketplace, ongoing or sustained drug innovation can provide the key competitive advantage for pharmaceutical companies to maintain the relative shares of the market. Hence, explorative research and development (R\&D) plays an important role in the successful operations for pharmaceutical companies. They often pursue proactive $R \& D$ projects that take a lengthy time to develop and entail a continuous flow of knowledge creation and discrimination. However, investment decision-making of pharmaceutical research and development (R\&D) project is a complex and difficult task, since the discovery process of new drugs is a complex and challenging endeavor, and the project expenditure is extremely expensive and time consuming. R\&D managers often face the dilemma of either working continuously to develop a perfect drug or terminating the development process and delivering an acceptable drug. Specifically, the drug innovation process often takes on a significant level of inherent complexity, and the information obtained by decision-makers to decide whether to terminate the project is composed of scientific uncertainty (Zhao \& Chen, 2009). Because of these knowledge complexity, information uncertainty, and technology exploration during pharmaceutical R\&D process with long lead times for market and technology dynamics, the project managers that engage in pharmaceutical R\&D often confront to a huge challenge for assessing whether the drug development is worth undertaking (Chi etc., 1997). 
In such cases, the optimal stopping rule may help them deal with such difficult problems. The purpose of optimal stopping rule is to allocate the limited resources to pharmaceutical projects by a certain method (e.g., pursuing for the optimal stopping time as well as optimum levels of expenditures. In addition, it is important to keep away from financial losses while achieving long-term goals, in that expending too little may lead to reducing future returns, while expending too much could excessively consume company resources.

In classical R\&D models, the inter-arrival times are often assumed to be random variables which are exponentially distributed. For instance, Kamien and Schwartz derived the optimal timing of planned expenditures of R\&D projects in which the total effort required to complete the research satisfactorily is not known (Kamien \& Schwartz, 1971). Deshmukh and Chikte proposed that the project status changes stochastically due to the internal (technological) and the external (market) uncertainties (Deshmukh \& Chikte, 1977). Zuckerman derived the optimal investment strategies for an R\&D project in which the project status changes according to a non-homogeneous compound Poisson process by using diffusion approximation (Zuckerman, 1980). Posner and Zuckerman proposed that the optimal stopping strategy is a control limit policy under certain realistic conditions, in which the inter-arrival times between jumps are exponentially distributed (Posner \& Zuckerman, 1990). Spector and Zuckerman considered a stochastic R\&D model for a single firm operating in a competitive environment (Spector \& Zuckerman, 1997). Chao and Peck formulated a simple decision model to investigate the optimal funding level for an environmental $R \& D$ activity. The Bayesian approach is extended to situations where the researchers have prior beliefs different from the ultimate decision makers. In addition, the optimal research expenditure was sensitive to the technical difficulty of the research area (Chao \& Peck, 1999). Mehrez and David analyzed the problem of determining the timing of risky R\&D tasks (activities) and non-routine tasks through a simple non-linear mathematical programming model. The typical non-convex or non-concave structure of the objective function, which maximizes the expected discounted net value of the project, implies the possibility of multiple local optimal solutions. For practical purposes, the global solution can be identified either by employing global optimization methods satisfying the K-T necessary conditions (Mehrez \& David, 1999). Watanabe etc. considered a two-dimensional dynamic model of an investment process, which describes the change in the volume of production and the amount of accumulated investments. The Pontryagin maximum principle is used to solve the problem (Watanabe etc., 2001). Zhao and Chen investigated an optimal stopping model for pharmaceutical R\&D projects, in which each individual round of lead compound arrives according to a Poisson process (Zhao \& Chen, 2009).

Obviously, the inter-arrival times are usually regarded as random variables, and the net present value of R\&D project is described by probability theory in traditional literature. In summary, the optimal stopping decision of pharmaceutical R\&D project is a difficult problem, for which many comprehensive models and solution technologies have been developed in order to deal with its complexity. In this paper, we present the dynamic, random fuzzy R\&D decision model that allows the decision-maker to "sell" the obtained technology at any point of time and treats technology as a key determinant during the pharmaceutical R\&D process. The R\&D managers should make stopping decisions based on the quality of the best scientific discoveries at hand and on the remaining time still allowed the project continuing. Specifically, three types of optimal stopping models with flexible termination time are introduced according to different decision objectives, and the inter-arrival times between jumps and jump magnitudes are all treated as random fuzzy variables with arbitrary distributions. Furthermore, the stop time is incorporated into the pharmaceutical R\&D project as decision variable by allowing the decision-maker to sell the obtained technology. To solve the proposed models, the simultaneous perturbation stochastic approximation (SPSA) algorithm based on random fuzzy simulation is designed to obtain the optimal stopping time of the pharmaceutical R\&D project. Finally, the numerical examples presented in the end of this paper show that these models are widely applicable.

The rest of this paper is organized as follows. In Section 2, we first give some basic concepts and arithmetic of fuzzy variables and random fuzzy variables. In section 3 , we focus our attention on the detail of the pharmaceutical R\&D optimal stopping decision problem to motivate our random fuzzy programming models. Section 4 presents three types of random fuzzy programming models for pharmaceutical R\&D optimal stopping decision. Section 5 proposes the random fuzzy simulation techniques to estimate the expected return, the $\alpha$-optimistic return and the chance function of return event which are utilized in Section 6 to obtain the objective functions of programming models. To solve the proposed models, Section 7 introduces a hybrid intelligent algorithm which integrates stochastic simulation and SPSA algorithm. After that, Section 8 provides a detailed examination through numerical examples to demonstrate the strength of our proposed models. The last section summarizes the paper. 


\section{Preliminaries}

In this section, some basic concepts and arithmetic of random fuzzy variables will be introduced as follows. The interested readers may refer to (Liu, 2002) and (Liu \& Liu, 2003) about more concepts and important properties of random fuzzy theory.

\subsection{Random Fuzzy Variables}

Definition 1. (Nahmias, 1978) Let $\Theta$ be a nonempty set, and $\mathcal{P}(\Theta)$ the power set of $\Theta$ (i.e., $\mathcal{P}(\Theta)$ is the collection of all subsets of $\Theta)$. For each $A \in \mathcal{P}(\Theta)$, the nonnegative number $\operatorname{Pos}\{A\}$ is called its possibility measure, such that

(1) $\operatorname{Pos}\{\emptyset\}=0, \operatorname{Pos}(\Theta)=1$;

(2) $\operatorname{Pos}\left\{\bigcup_{k} A_{k}\right\}=\sup _{k} \operatorname{Pos}\left\{A_{k}\right\}$ for any collection $\left\{A_{k}\right\}$ in $\mathcal{P}(\Theta)$.

The function Pos is called a possibility measure, and the triplet $(\Theta, \mathcal{P}(\Theta)$, Pos) is called a possibility space.

Definition 2. (Liu, 2002) Suppose that $\left(\Theta_{i}, \mathcal{P}\left(\Theta_{i}\right), \mathrm{Pos}_{i}\right), i=1,2, \ldots, n$ are possibility spaces. Write $\Theta=\Theta_{1} \times$ $\Theta_{2} \times \cdots \times \Theta_{n}$ and $\operatorname{Pos}=\operatorname{Pos}_{1} \wedge \operatorname{Pos}_{2} \wedge \cdots \wedge \operatorname{Pos}_{n}$. Then the set function $\operatorname{Pos} i$ s a possibility measure on $\mathcal{P}(\Theta)$, and $(\Theta, \mathcal{P}(\Theta)$, Pos $)$ is called a product possibility space of $\left(\Theta_{i}, \mathcal{P}\left(\Theta_{i}\right), \mathrm{Pos}_{i}\right), i=1,2, \ldots, n$. Here, the symbol $\wedge$ means the minimum.

Definition 3. (Liu, 2004) Let $\left(\Theta_{i}, \mathcal{P}\left(\Theta_{i}\right), \operatorname{Pos}_{i}\right), i=1,2, \ldots$ be an arbitrary sequence of possibility spaces and $\Theta=\Theta_{1} \times \Theta_{2} \times \cdots$. Define Pos on $\mathcal{P}(\Theta)$ such that

$$
\operatorname{Pos}\{A\}=\sup _{\left(\theta_{1}, \theta_{2}, \ldots\right) \in A} \operatorname{Pos}_{1}\left\{\theta_{1}\right\} \wedge \operatorname{Pos}_{2}\left\{\theta_{2}\right\} \wedge \cdots
$$

Then the set function Pos is a possibility measure on $\mathcal{P}(\Theta)$, and $(\Theta, \mathcal{P}(\Theta)$, Pos) is a product possibility space of $\left(\Theta_{i}, \mathcal{P}\left(\Theta_{i}\right), \operatorname{Pos}_{i}\right), i=1,2, \ldots$

Definition 4. (Liu, 2002) A fuzzy variable $\xi$ is defined as a mapping function from the possibility space $(\Theta, \mathcal{P}(\Theta)$, Pos) to the set of real numbers $\mathcal{R}$.

Definition 5. (Liu, 2002) Let $\xi$ be a fuzzy variable on the possibility space $(\Theta, \mathcal{P}(\Theta)$, Pos), $x \in \mathcal{R}$. Then its membership function is derived from the possibility measure by

$$
\mu(x)=\operatorname{Pos}\{\theta \in \Theta \mid \xi(\theta)=x\} .
$$

Example 1. By a triangular fuzzy variable, we mean the fuzzy variable fully determined by the triplet $\left(r_{1}, r_{2}, r_{3}\right)$ of crisp numbers with $r_{1}<r_{2}<r_{3}$, whose membership function is expressed by

$$
\mu(x)=\left\{\begin{array}{cl}
\frac{x-r_{1}}{r_{2}-r_{1}}, & \text { if } r_{1} \leq x \leq r_{2} \\
\frac{x-r_{3}}{r_{2}-r_{3}}, & \text { if } r_{2} \leq x \leq r_{3} \\
0, & \text { otherwise. }
\end{array}\right.
$$

Remark 1. A fuzzy variable $\xi$ can represent some unknown information. For a given real number $x, \mu(x)$ means the possibility of the event that $\xi$ is equal to $x$. It can be used to depict the real economic phenomena. For example, the return caused by an uncertain jump event of R\&D project and known roughly can be described as a fuzzy variable. Then $\mu(x)$ will represent the possibility that the real return is equal to $x$.

On the basis of the possibility measure Pos, the necessity measure (denoted by Nec) and credibility measure (denoted by Cr) of fuzzy event can be represented as follows.

Definition 6. (Liu, 2002) Let $\left(\Theta, \mathcal{P}(\Theta)\right.$, Pos) be a possibility space, A a set in $\mathcal{P}(\Theta)$, and $A^{c}$ the opposite set of $A$. Then the necessity measure of $A$ is defined by

$$
\operatorname{Nec}\{A\}=1-\operatorname{Pos}\left\{A^{c}\right\} .
$$

Definition 7. (Liu $\mathcal{F}$ Liu, 2002) Let $(\Theta, \mathcal{P}(\Theta)$, Pos) be a possibility space, and A a set in $\mathcal{P}(\Theta)$. Then the credibility measure of $A$ is defined by

$$
\operatorname{Cr}\{A\}=\frac{1}{2}(\operatorname{Pos}\{A\}+\operatorname{Nec}\{A\}) .
$$


Definition 8. (Liu $\mathcal{E}$ Liu, 2002) Let $\xi$ be a fuzzy variable. The expected value $E[\xi]$ is defined as

$$
E[\xi]=\int_{0}^{+\infty} \operatorname{Cr}\{\xi \geq r\} \mathrm{d} r-\int_{-\infty}^{0} \operatorname{Cr}\{\xi \leq r\} \mathrm{d} r
$$

provided that at least one of the two integrals is finite.

Definition 9. (Liu, 2002) Let $\xi$ be a fuzzy variable and $\alpha \in(0,1]$. Then

$$
\xi_{\alpha}^{\prime}=\inf \{r \mid \operatorname{Pos}\{\xi \leq r\} \geq \alpha\} \quad \text { and } \quad \xi_{\alpha}^{\prime \prime}=\sup \{r \mid \operatorname{Pos}\{\xi \geq r\} \geq \alpha\}
$$

are called the $\alpha$-pessimistic value and the $\alpha$-optimistic value of $\xi$, respectively.

Proposition 1. (Liu $\mathcal{E}$ Liu, 2002) Let $\xi$ be a fuzzy variable with the finite expected value $E[\xi]$. Then we have

$$
E[\xi]=\frac{1}{2} \int_{0}^{1}\left(\xi_{\alpha}^{\prime}+\xi_{\alpha}^{\prime \prime}\right) \mathrm{d} \alpha
$$

Furthermore, Liu presented the concept of random fuzzy variable to depict the scenario where the randomness and fuzziness appear simultaneously (Liu, 2002). Random fuzzy variable can be regarded as a natural extension to fuzzy variable. The concept of random fuzzy variable is presented as follows.

Definition 10. (Liu, 2002) A random fuzzy variable $\xi$ is defined as a function from the possibility space $(\Theta, \mathcal{P}(\Theta)$, Pos) to a collection of random variables.

Example 2. Let $\xi \sim \mathrm{N}(v, 1)$, where $v$ is a fuzzy variable with membership function $\mu_{v}(x)=[1-|x-2|] \vee 0$. Then $\xi$ is a random fuzzy variable taking "normally distributed variable $N(v, 1)$ " values. Here, the symbol $\vee$ means the maximum.

Definition 11. (Liu, 2002) Let $\xi_{i}$ be random fuzzy variables on the possibility spaces $\left(\Theta_{i}, \mathcal{P}\left(\Theta_{i}\right), \operatorname{Pos}_{i}\right), i=$ $1,2, \ldots, n$, respectively, and $f: \mathfrak{R}^{n} \rightarrow \mathfrak{R}$ a measurable function. Then $\xi=f\left(\xi_{1}, \xi_{2}, \ldots, \xi_{n}\right)$ is a random fuzzy variable on the product possibility space $(\Theta, \mathcal{P}(\Theta)$, Pos), defined as

$$
\xi\left(\theta_{1}, \theta_{2}, \ldots, \theta_{n}\right)=f\left(\xi_{1}\left(\theta_{1}\right), \xi_{2}\left(\theta_{2}\right), \ldots, \xi_{n}\left(\theta_{n}\right)\right)
$$

for all $\left(\theta_{1}, \theta_{2}, \ldots, \theta_{n}\right) \in \Theta$.

\subsection{Random Fuzzy Arithmetic}

Definition 12. (Liu, 2002) A random fuzzy variable $\xi$ is named to be nonnegative if and only if for each $\theta \in \Theta$ with $\operatorname{Pos}\{\theta\}>0, \operatorname{Pr}\{\xi(\theta)<0\}=0$.

Definition 13. (Liu $\mathcal{E}$ Liu, 2003) Let $\xi$ be a random fuzzy variable defined on the possibility space $(\Theta, \mathcal{P}(\Theta)$, Pos). Then the expected value operator $E[\xi]$ is defined by

$$
E[\xi]=\int_{0}^{+\infty} \operatorname{Cr}\{\theta \in \Theta \mid E[\xi(\theta)] \geq r\} \mathrm{d} r-\int_{-\infty}^{0} \operatorname{Cr}\{\theta \in \Theta \mid E[\xi(\theta)] \leq r\} \mathrm{d} r
$$

on the condition that at least one of the two integrals is finite.

Remark 2. If the random fuzzy variable $\xi$ degenerates to a random variable, then the expected value varies as follows

$$
E[\xi]=\int_{0}^{+\infty} \operatorname{Pr}\{\xi \geq r\} \mathrm{d} r-\int_{-\infty}^{0} \operatorname{Pr}\{\xi \leq r\} \mathrm{d} r
$$

which is the traditional mathematical expectation of the random variable $\xi$. If the random fuzzy variable $\xi$ degenerates to a fuzzy variable, then the expected value becomes

$$
E[\xi]=\int_{0}^{+\infty} \operatorname{Cr}\{\xi \geq r\} \mathrm{d} r-\int_{-\infty}^{0} \operatorname{Cr}\{\xi \leq r\} \mathrm{d} r
$$

which is the expected value of the fuzzy variable $\xi$ mentioned above. 
Definition 14. ( $\mathrm{Li} \mathcal{E} \mathrm{Liu}, 2006$ ) Random fuzzy variables $\xi_{1}, \xi_{2}, \ldots, \xi_{n}$ are named to be independent and identically distributed (iid) if and only if

$$
\operatorname{Pr}\left\{\xi_{i}(\theta) \in B_{1}\right\}, \operatorname{Pr}\left\{\xi_{i}(\theta) \in B_{2}\right\}, \ldots, \operatorname{Pr}\left\{\xi_{i}(\theta) \in B_{m}\right\}, i=1,2, \ldots, n
$$

are iid fuzzy vectors for any Borel sets $B_{1}, B_{2}, \ldots, B_{m}$ of $\mathcal{R}$ and any positive integer $m$.

Proposition 2. Assume that $\xi$ and $\eta$ are two random fuzzy variables with finite expected values. If $E[\xi(\theta)]$ and $E[\eta(\theta)]$ are independent fuzzy variables, then

$$
E[a \xi+b \eta]=a E[\xi]+b E[\eta]
$$

for any real numbers $a$ and $b$.

Definition 15. (Liu, 2002) Let $\xi$ be a random fuzzy variable, any set $A \in \mathcal{P}(\Theta)$, and $B$ a Borel set of $\mathcal{R}$. Then the chance of random fuzzy event $\xi \in B$ is a function from $(0,1]$ to $[0,1]$, defined as

$$
\operatorname{Ch}\{\xi \in B\}(\alpha)=\sup _{\operatorname{Cr}\{A\} \geq \alpha} \inf _{\theta \in A} \operatorname{Pr}\{\xi(\theta) \in B\} .
$$

\section{Random Fuzzy Process for Pharmaceutical R\&D Project}

The pharmaceutical industry is uniquely characterized by high exposure to technical and market risks and long development duration. The R\&D process can be seen as a diffusion jump process in which the project status changes continuously (Posner \& Zuckerman, 1990). In this study, we examine a pharmaceutical R\&D project under random fuzzy environments, in which the project status can be evaluated at any point in time. Furthermore, the project status can be measured by the relative drug quality developed so far in comparison with other competing drugs currently in the market. The project reward depends on its final status of drug quality. Random fuzzy variables can be used model the technological and market uncertainties during the drug development process.

The random fuzzy decision model can be formally formulated as follows. Let $\{X(t) ; t \geq 0\}, X(0)=0$, be a onesided, non-decreasing random fuzzy diffusion process. The project status $X(t)$ can be interpreted as the monetary value of knowledge accumulated by the research program up to time $t$. Moreover, a progress in the project status corresponds to a scientific breakthrough or a technological discovery. Let $T_{i}$ denote the inter-arrival times between the $(i-1)$ th and $i$ th breakthrough, $i=1,2, \ldots$, respectively. $\left\{T_{i}, i \geq 1\right\}$ are defined as a set of iid bounded nonnegative random fuzzy variables defined on the possibility spaces $\left(\Theta_{1 i}, \mathcal{P}\left(\Theta_{1 i}\right), \operatorname{Pos}_{1 i}\right), i=1,2, \ldots$, respectively, and $F(\cdot)$ represents the same distribution function with fuzzy parameters. Let $S_{n}$ represent the arrival time of the $n$th breakthrough, $S_{0}=0$, then

$$
S_{n}=\sum_{i=1}^{n} T_{i} .
$$

The process $\left\{S_{n}, n \geq 1\right\}$ becomes a random fuzzy renewal process on the possibility space $\left(\Theta_{1}, \mathcal{P}\left(\Theta_{1}\right)\right.$, Pos 1$)$ which is the product possibility space of $\left(\Theta_{1 i}, \mathcal{P}\left(\Theta_{1 i}\right), \operatorname{Pos}_{1 i}\right), i=1,2, \ldots$

Let $N_{t}$ denote the number of jumps in $(0, t]$. Then

$$
N_{t}=\sup \left\{n: S_{n} \leq t\right\} .
$$

Obviously, $N_{t}$ is also a random fuzzy variable on the possibility space $\left.\left(\Theta_{1}, \mathcal{P}\left(\Theta_{1}\right) \text {, Pos }\right)_{1}\right)$. For any fixed $\boldsymbol{\theta}_{1}=$ $\left(\theta_{11}, \theta_{12}, \ldots\right) \in \Theta_{1}, N_{t}\left(\boldsymbol{\theta}_{1}\right)$ will become a random variable with the probability distribution

$$
\operatorname{Pr}\left\{N_{t}\left(\boldsymbol{\theta}_{1}\right)=n\right\}=\operatorname{Pr}\left\{S_{n}\left(\boldsymbol{\theta}_{1}\right) \leq t\right\}-\operatorname{Pr}\left\{S_{n+1}\left(\boldsymbol{\theta}_{1}\right) \leq t\right\}, \quad n=0,1,2, \ldots,
$$

where $S_{n}\left(\boldsymbol{\theta}_{1}\right)=\sum_{i=1}^{n} T_{i}\left(\theta_{1 i}\right)$ and $S_{n+1}\left(\boldsymbol{\theta}_{1}\right)=\sum_{i=1}^{n+1} T_{i}\left(\theta_{1 i}\right)$.

Furthermore, the jump magnitudes $Y_{i}$ associated with the drug development process are assumed to be iid bounded nonnegative random fuzzy variables defined on the possibility spaces $\left(\Theta_{2 i}, \mathcal{P}\left(\Theta_{2 i}\right), \operatorname{Pos}_{2 i}\right), i=1,2, \ldots$, respectively, which have the same distribution function $G(\cdot)$ with fuzzy parameters. Then the value of project status at time $t$ is given as follows according to the random fuzzy renewal theory (Zhao etc., 2006).

$$
X(t)=\sum_{i=1}^{N_{t}} Y_{i}
$$


$X(t)$ represents the reward value of project, which is accumulated by a set of activities (breakthroughs) completed successfully. Therefore, $X(t)$ is also a random fuzzy variable on the product possibility space $(\Theta, \mathcal{P}(\Theta)$, Pos) of $\left(\Theta_{1}, \mathcal{P}\left(\Theta_{1}\right), \operatorname{Pos}_{1}\right)$ and $\left(\Theta_{2}, \mathcal{P}\left(\Theta_{2}\right), \operatorname{Pos}_{2}\right)$, in which $\left(\Theta_{2}, \mathcal{P}\left(\Theta_{2}\right), \operatorname{Pos}_{2}\right)$ is the product possibility space of $\left(\Theta_{2 i}, \mathcal{P}\left(\Theta_{2 i}\right)\right.$, $\left.\operatorname{Pos}_{2 i}\right), i=1,2, \ldots$ Here, the sequences $\left\{T_{i}, i \geq 1\right\}$ and $\left\{Y_{i}, i \geq 1\right\}$ are assumed to be independent with each other. Thus, the random fuzzy renewal number $N_{t}$ is independent of the random fuzzy renewal magnitudes $Y_{i}$.

Remark 3. The independence between the jump number and the magnitude of new discoveries is a classical assumption in R\&D literature. We consider a drug development project in which an increase in expenditures is reflected in an increase in the number of research groups working on the singular project, with each group employing a different scientific approach. In such case, the expenditure policy affects the arrival rate of new discoveries, but not necessarily their magnitude.

The project can be terminated at any time throughout the development process. Specifically, if an activity is completed, the manager has two alternative actions: either to continue the process to a new status by the next technological discovery, or to terminate the process and to obtain the terminal reward depending on the project status. We assume the stopping time is denoted by $T$, then $X(T)$ denote the final return from the project. So the discounted value of $X(T)$ is denoted by $\exp (-r T) X(T)$, where $r$ is an applicable continuous discount factor and denotes the cost of capital. Based on the above mentioned, the discounted net return from the project can be given as follows.

$$
\xi(T)=\exp (-r T) X(T)-\int_{0}^{T} C \exp (-r t) \mathrm{dt}-\mathrm{I} .
$$

where $C$ denotes a continuous flow of investment per unit of time during the drug development project, and $I$ represents an initial investment required to start the project. Note that in various pharmaceutical R\&D activities, in order to avoid the high cost associated with hiring and firing of highly qualified research staff and engineers, pharmaceutical R\&D projects are generally operated at a constant expenditure (Spector \& Zuckerman, 1997). Obviously, $\xi(T)$ is also a random fuzzy variable defined on the product possibility space $(\Theta, \mathcal{P}(\Theta)$, Pos) because it is a function of random fuzzy variable $X(T)$.

Remark 4. The investment cost $C$ can be viewed as the opportunity cost of the resource expenditure (particularly the engineering technology personnel) invested into the pharmaceutical R\&D project. Otherwise, the main factor determining the total cost was the stopping time which determines when the pharmaceutical R\&D project should be terminated. Furthermore, it should be noted that $I$ becomes a sunk investment after the initiation of the pharmaceutical R\&D project and thus has no bearing on the optimal stopping time for an ongoing project, although this initial investment does affect the decision on whether to undertake the project in the beginning (Chi etc., 1997).

\section{Random Fuzzy Decision Models}

In pharmaceutical industry, the impact of uncertain factors on the optimal stopping decision is crucial and complex. In such an uncertain and randomly evolving situation, the manager often faces serious difficulty in assessing whether the project is still worth continuing because established methods for project appraisal do not lend themselves to a dynamic evaluation of the project. The mathematic programming technology is a powerful tool to these decision problems for different management objectives. Therefore, the random fuzzy programming technology is used to deal with the optimal stopping decision in this section. The interested readers may consult (Liu, 2002) about the random fuzzy programming issues. Given the specification outlined above, three types of pharmaceutical R\&D decision models including expected value model, chance-constrained programming model and dependent-chance programming model are presented as follows. These decision models can be applied in not only pharmaceutical R\&D project but also the risk investment projects.

\subsection{Random Fuzzy Expected Value Model}

If the project manager is risk neutral, which is the commonly used optimality criterion to model the R\&D decision problems under uncertain environments, the decision objective is to obtain an optimal stopping time $T^{*}$ that maximizes the expected discounted net return from the project under certain cost constraints in a finite time-horizon, then the random fuzzy expected value model (EVM) can be presented as follows:

$$
\left\{\begin{array}{l}
\max E[\xi(T)] \\
\text { subject to: } \\
T, C, I>0 \\
0<r<1 .
\end{array}\right.
$$


Proposition 3. If the random fuzzy variable $\xi$ degenerates to a random variable, then the expected value in the above EVM becomes

$$
E[\xi(T)]=\int_{0}^{+\infty} \operatorname{Pr}\{\xi(T) \geq r\} \mathrm{d} r-\int_{-\infty}^{0} \operatorname{Pr}\{\xi(T) \leq r\} \mathrm{d} r,
$$

which is the general expectation concepts of the random variable $\xi(T)$.

Proposition 4. If the random fuzzy variable $\xi$ degenerates to a fuzzy variable, then the expected value in the above EVM becomes

$$
E[\xi(T)]=\int_{0}^{+\infty} \operatorname{Cr}\{\xi(T) \geq r\} \mathrm{d} r-\int_{-\infty}^{0} \operatorname{Cr}\{\xi(T) \leq r\} \mathrm{d} r,
$$

which is the expected value operator of the fuzzy variable $\xi(T)$.

\subsection{Random Fuzzy Chance-constrained Programming Model}

Chance-constrained programming which is presented by (Charnes \& Cooper, 1959) as a new modelling philosophy, can be often applied to solve the practical optimization problems on the condition that chance constraints should hold with at least some given confidence levels. If the decision-maker wishes to maximize the final return in which the constraints hold with some predetermined confidence levels, we may employ the following chance-constrained programming model (CCPM):

$$
\left\{\begin{array}{l}
\max \bar{\xi} \\
\text { subject to: } \\
\quad \operatorname{Ch}\{\xi(\mathrm{T}) \geq \bar{\xi}\}(\alpha) \geq \beta \\
T, C, I>0 \\
0<r<1
\end{array}\right.
$$

where $\bar{\xi}$ is the $(\alpha, \beta)$-return which is defined as the largest value $\bar{\xi}$ satisfying the condition of $\operatorname{Ch}\{\xi(\mathrm{T}) \geq \bar{\xi}\}(\alpha) \geq \beta$. Here, $\alpha$ and $\beta$ are the predetermined confidence level provided as an appropriate safety margin by the decisionmaker with $0<\alpha<1$ and $0<\beta<1$, respectively.

\subsection{Random Fuzzy Dependent-chance Programming Model}

The pharmaceutical $R \& D$ project is often required to obtain the return within a fixed range $\left[0, \xi_{0}\right]$, where $\xi_{0}$ is the fixed terminal reward. If the decision-maker want to maximize the chance of satisfying the event $\left\{\xi(T) \geq \xi_{0}\right\}$ subject to an uncertain environment, then the dependent-chance programming model (DCPM) can be presented as follows:

$$
\left\{\begin{array}{l}
\max \operatorname{Ch}\left\{\xi(\mathrm{T}) \geq \xi_{0}\right\}(\alpha) \\
\text { subject to: } \\
T, C, I>0 \\
0<r<1
\end{array}\right.
$$

Remark 5. A pharmaceutical R\&D project is usually required to obtain a fixed terminal reward $\xi_{0}$ as the decision objective. The decision-maker may want to maximize the chance function satisfying the event $\left\{\xi(T) \geq \xi_{0}\right\}$. The DCPM models this type of investment decision problem. Specifically, DCPM replaces the concept of feasible set constraints as uncertain environment.

\section{Random Fuzzy Simulation}

In the random fuzzy decision models mentioned above in Section 4 , the three types of reward performance with random fuzzy variables as the expected return $E[\xi(T)]$, the $(\alpha, \beta)$-return, and the chance function $\mathrm{Ch}\left\{\xi(\mathrm{T}) \geq \xi_{0}\right\}(\alpha)$. These uncertain functions cannot be calculated through the analytic algorithm in general. Therefore, we will simulate these uncertain functions by random fuzzy simulations according to the concepts of chance measure and expected value of random fuzzy variable.

\subsection{Random Fuzzy Simulation for $E[\xi(T)]$}

For any given termination policy $T$, for the purpose of calculating the expected return $E[\xi(T)]$ from the project completion, we randomly sample $\theta_{1}, \theta_{2}, \ldots, \theta_{N}$ from the universe $\Theta$ such that $\operatorname{Pos}\left\{\theta_{i}\right\} \geq \delta, i=1,2, \ldots, N$, where $\delta$ 
is a sufficiently small number. We can estimate $E\left[\xi\left(\theta_{i}\right)\right]$ by the stochastic simulation technology, in that $\xi\left(\theta_{i}\right)$ take on random variables for fixed $\theta_{i}, i=1,2, \ldots, N$. Then the random fuzzy simulation procedure for $E[\xi(T)]$ is given as follows.

Step 1. Set $L=0$.

Step 2. Randomly sample sequences $\theta_{i}$ from the universe $\Theta$ such that $\operatorname{Pos}\left\{\theta_{i}\right\} \geq \delta, i=1,2, \ldots, N$, where $N$ is a sufficiently large number.

Step 3. Let $a=\min _{1 \leq j \leq N} E\left[\xi\left(\theta_{j}\right)\right]$ and $b=\max _{1 \leq j \leq N} E\left[\xi\left(\theta_{j}\right]\right.$, in which $E\left[\xi\left(\theta_{j}\right)\right]$ can be estimated by stochastic simulation for $j=1,2, \ldots, N$.

Step 4. Randomly generate $r$ from $[a, b]$. If $r \geq 0$, then $L \leftarrow L+\operatorname{Cr}\{\theta \in \Theta \mid E[\xi(\theta] \geq r\}$. If $r<0$, then $L \leftarrow L-\operatorname{Cr}\{\theta \in \Theta \mid E[\xi(\theta] \leq r\}$.

Step 5. Repeat the fourth step for $N$ times.

Step 6. $E[\xi(T)]=a \vee 0+b \wedge 0+L \cdot(b-a) / N$.

\subsection{Random Fuzzy Simulation for $(\alpha, \beta)$-return}

For any given stopping policy $T$ which determines when the project should be terminated, and any confidence levels $\alpha$ and $\beta$, we first set $\bar{\xi}=0$ in order to obtain the maximal $\bar{\xi}$ such that $\operatorname{Ch}\{\xi(T) \geq \bar{\xi}\}(\alpha) \geq \beta$. Randomly generate a crisp point $\theta$ from the universe $\Theta$ such that $\operatorname{Pos}\{\theta\} \geq \alpha$. Hence, $\xi(\theta)$ become a random variable. For $\xi^{\prime}$ such that $\operatorname{Pr}\left\{\xi(\theta) \geq \xi^{\prime}\right\} \geq \beta$ by stochastic simulation technology, we set $\bar{\xi}=\xi^{\prime}$ provided that $\bar{\xi}<\xi^{\prime}$. Then the simulation procedure for $(\alpha, \beta)$-return can be generalized as follows.

Step 1. Set $\bar{\xi}=0$.

Step 2. Generate a crisp point $\theta$ from the universe $\Theta$ such that $\operatorname{Pos}\{\theta\} \geq \alpha$.

Step 3. Find the maximal $\xi^{\prime}$ such that $\operatorname{Pr}\left\{\xi(\theta) \geq \xi^{\prime}\right\} \geq \beta$.

Step 4. Set $\bar{\xi}=\xi^{\prime}$ on the condition of $\bar{\xi}<\xi^{\prime}$.

Step 5. Repeat the second step to fourth step for $N$ times.

Step 6. Return $\bar{\xi}$.

\subsection{Random Fuzzy Simulation for Chance}

For any given decision variable $T$ and the confidence level $\alpha$, we tend to simulate the chance $\operatorname{Ch}\left\{\xi(T) \geq \xi_{0}\right\}(\alpha)$. The simulation for chance can be run as follows.

Step 1. Set $L=0$.

Step 2. Generate a crisp point $\theta$ from the universe $\Theta$ such that $\operatorname{Pos}\{\theta\} \geq \alpha$.

Step 3. Compute the probability $\operatorname{Pr}\left\{\xi(\theta) \geq \xi_{0}\right\}$ by the stochastic simulation.

Step 4. Set $L=\operatorname{Pr}\left\{\xi(\theta) \geq \xi_{0}\right\}$ provided that $L<\operatorname{Pr}\left\{\xi(\theta) \geq \xi_{0}\right\}$.

Step 5. Repeat the second step to fourth step for $N$ times.

Step 6. Return $L$.

\section{Hybrid Intelligent Algorithm}

To find an optimal stopping strategy for pharmaceutical R\&D project under uncertainty, we need to design some heuristic algorithm to solve the proposed models in Section 4. SPSA algorithm is a particular multivariate stochastic approximation technique which requires only two noisy measurements of objective function per iteration and 
shows fast convergence in practice, regardless of the dimension of the optimization problem. Hence, it is applicable in both the stochastic gradient and gradient-free settings and well suitable for problems with a large number of variables to be optimized (Spall, 2003). In this section, we will design a hybrid intelligent algorithm by employing the random fuzzy simulations which can estimate the above three types of random fuzzy functions into the SPSA algorithm. The hybrid intelligent algorithm can be presented step-by-step as follows.

Step 1. Initialization and coefficient selection. Set the counter index $k=0$. Choose an initial guess $\hat{T}_{0}$ and nonnegative parameters $a, c, A, \alpha$, and $\gamma$ in the SPSA, then obtain two sequences $a_{k}=a /(A+k+1)^{\alpha}$ and $c_{k}=c /(k+1)^{\gamma}$. Practically effective (or theoretically valid) values for $\alpha$ and $\gamma$ are 0.602 and 0.101 , respectively. $a, c$, and $A$ can be determined based on the practical guidelines.

Step 2. Generation of the simultaneous perturbation vector. Generate a $p$-dimensional random perturbation vector $\boldsymbol{\Delta}_{k}$, where each of the $p$ components of $\boldsymbol{\Delta}_{k}$ are independently generated from a zero-mean probability distribution satisfying the conditions above. An effective (or theoretically valid) choice for each components of $\boldsymbol{\Delta}_{k}$ is to be obtained by a symmetric Bernoulli \pm 1 distribution with probability of $1 / 2$ for each \pm 1 outcomes, although other choices are valid and may be desirable in some applications.

Step 3. Loss function evaluations. Obtain two measurements of the loss function based on the simultaneous perturbation around the current $\hat{T}_{k}: y\left(\hat{T}_{k}+c_{k} \Delta_{k}\right)$ and $y\left(\hat{T}_{k}-c_{k} \Delta_{k}\right)$ with the $c_{k}$ and $\Delta_{k}$ from steps 1 and 2.

Step 4. Gradient approximation. Generate the simultaneous perturbation approximation to the unknown gradient $\hat{g}_{k}\left(\hat{T}_{k}\right)$ as follows:

$$
\hat{g}_{k}\left(\hat{T}_{k}\right)=\frac{y\left(\hat{T}_{k}+c_{k} \boldsymbol{\Delta}_{k}\right)-y\left(\hat{T}_{k}-c_{k} \boldsymbol{\Delta}_{k}\right)}{2 c_{k}}\left[\begin{array}{c}
\Delta_{k 1}^{-1} \\
\Delta_{k 2}^{-1} \\
\vdots \\
\Delta_{k p}^{-1}
\end{array}\right] .
$$

It is sometimes useful to average several gradient approximations at $\hat{T}_{k}$, each formed from an independent generation of $\boldsymbol{\Delta}_{k}$.

Step 5. Update $T$ estimation. Update $\hat{T}_{k}$ to a new value $\hat{T}_{k+1}$ using the standard form of stochastic approximation recursion $\hat{T}_{k+1}=\hat{T}_{k}-a_{k} \hat{g}_{k}\left(\hat{T}_{k}\right)$. Check for constraint violation (if relevant) and modify the updated $T$. An effective way to handle constraints is to simply map violating elements of $T$ to the nearest valid point.

Step 6. Iteration or termination. Return to Step 2 with $k+1$ replacing $k$. Terminate the process if there is little change in several successive iterates or if the maximum allowable number of iterations has been reached.

\section{Numerical Example}

In this section, some numerical examples are presented to illustrate the feasibility of the modelling and the effectiveness of the hybrid intelligent algorithm.

For a pharmaceutical $\mathrm{R} \& \mathrm{D}$ project, the inter-arrival times $T_{i}$ are iid random fuzzy variables which observe the exponential distribution $T_{i} \sim \mathcal{E} \mathcal{X P}\left(\lambda_{i}\right)$ whose parameters are triangular fuzzy variables $\lambda_{i}=(1,2,3)$. So the jump numbers $N_{t}$ observe Poisson distribution. Furthermore, the jump magnitudes $Y_{i}$ are iid lognormally distributed random fuzzy variables defined as

$$
Y_{i} \sim \operatorname{LOGN}\left(\mu_{\mathrm{i}}, \sigma_{\mathrm{i}}^{2}\right), \quad \text { with } \quad \mu_{\mathrm{i}}=(1,2,3), \sigma_{\mathrm{i}}^{2}=(0,1,2), \mathrm{i}=1,2, \ldots
$$

The continuous discount factor is taken by $r=0.10$. Therefore, the project status $X(t)$ at time $t$ can be obtained by random fuzzy simulation. Otherwise, the R\&D manager may be able to compute the continuous flow cost fairly accurately according to the types and amounts of resources (e.g., personnel, equipment and materials). Here, we assume that the constant cost per unit of time of operating the projet is $C=10$, and the initial investment is $I=10$.

Example 1. For the random fuzzy expected value model, we consider an initial condition that the parameter $\hat{T}_{0}=0.5$ and select coefficients $a=0.10, c=0.05, A=10, \alpha=0.602, \gamma=0.101$ of the SPSA algorithm. Then we start the iteration process of the SPSA algorithm embedding the random fuzzy simulation. Finally, a run of the hybrid intelligent algorithm (2000 cycles in random fuzzy simulation, 6000 iterations in SPSA algorithm) shows that the optimal stopping time is $T^{*}=5.5372$, and the expected discounted net return from the project completion is 28.0508. Fig. 1 shows the variation of optimal solution for the stopping policy of pharmaceutical R\&D project. 


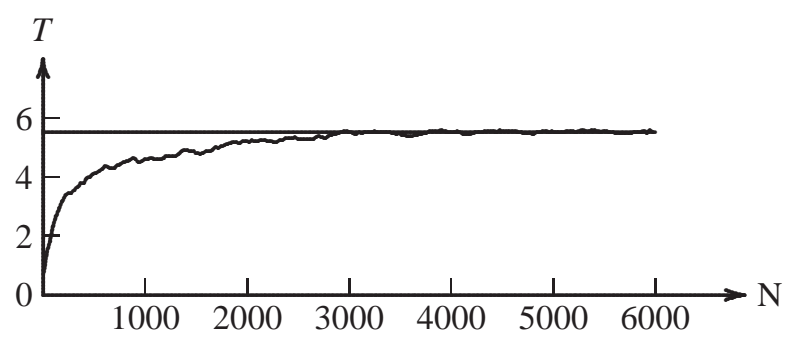

Figure 1. A Simulation Process for the Optimal Stopping Time in EVM

Example 2. For the random fuzzy chance-constrained programming model, we give the initial value $\hat{T}_{0}=0.5$. And the parameters of SPSA algorithm are presented as follows: $a=0.15, c=0.20, A=100, \alpha=0.602, \gamma=0.101$. Furthermore, the predetermined confidence levels are assumed as $\alpha=0.9$ and $\beta=0.8$. A run of the hybrid intelligent algorithm (2000 cycles in random fuzzy simulation, 6000 iterations in SPSA algorithm) shows that the optimal stopping time is $T^{*}=6.0518$, and the maximal $\alpha, \beta$-return is $\bar{\xi}=26.1551$. Fig. 2 shows the simulation process of the stopping policy for pharmaceutical R\&D project.

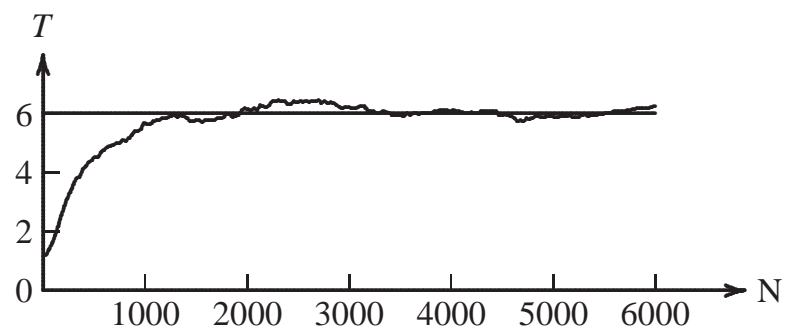

Figure 2. A Simulation Process for Stopping Time in CCPM

Example 3. For the random fuzzy dependent-chance programming model, we initialize the original value $\hat{T}_{0}=0.5$. And the optimization parameters of SPSA algorithm are given as follows: $a=0.16, c=0.20, A=100, \alpha=$ $0.602, \gamma=0.101$. Furthermore, the confidence level is assumed as $\alpha=0.9$ and the predetermined objective value $\xi_{0}$ is 25 . A run of the hybrid intelligent algorithm (2000 cycles in random fuzzy simulation, 6000 iterations in SPSA algorithm) shows the optimal solution is $T^{*}=5.7643$ with the chance $\operatorname{Ch}\{\xi(T) \geq 25\}(0.9)=0.8743$. Fig. 3 depicts a simulation process for the stopping strategy of pharmaceutical $R \& D$ project.

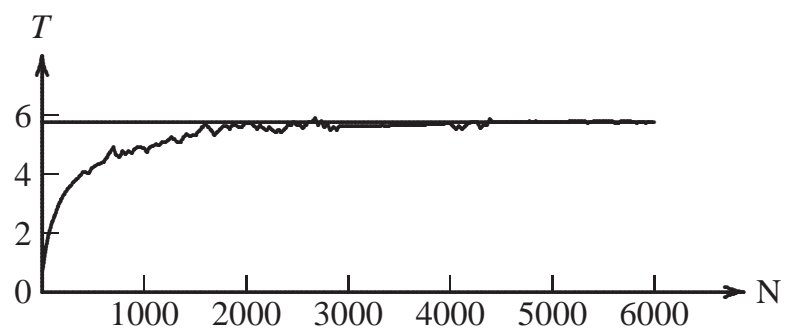

Figure 3. A Simulation Process for Stopping Time in DCPM

\section{Conclusions}

In this paper, we consider the pharmaceutical R\&D decision problems for different decision objects through random fuzzy programming methods. Specifically, we assume the knowledge accumulation process of pharmaceutical $R \& D$ project is a random fuzzy jump process. Every jump represents a scientific breakthrough or a new technology discovery. Furthermore, the termination time of the project completion is incorporated into the investment decision models as a decision variable by allowing the decision-maker to "sell" the obtained technology from the 
project at any point of time. The proposed model framework extends the classical approach in the R\&D literature from random environment to random fuzzy setting. As the numerical examples show that these models hold under the different objective function forms identified, they seem to possess considerable flexibility for different decision situations. Therefore, the advantages of these models include application in different type of pharmaceutical R\&D projects, enabling the decision-makers to integrate their discovery process and save expenditures. In future research we intend to further investigate other applications and introduce economic resource allocation into the pharmaceutical R\&D optimal stopping decision models.

\section{Acknowledgements}

This work was partially supported by a grant from the Humanities and Social Sciences Foundation of the Minis-try of Education (10YJC630352), a grant from the Humanities and Social Sciences Major Program of Anhui Province (SK2014ZD018), and a grant from the Jinglong Company Research Project (RD13210009). The author would like to thank the anonymous referees for helpful and constructive comments.

\section{References}

Chao, H., \& Peck, S. (1999). A decision model for environmental R\&D. Environment International, 25(6-7), 871-886. http://dx.doi.org/10.1016/S0160-4120(99)00063-X

Charnes, A., \& Cooper, W. (1959). Chance-constrained programming. Management Science, 6(1), 73-79. http://dx.doi.org/10.1287/mnsc.6.1.73

Chi, T., Liu, J., \& Chen, H. (1997). Optimal Stopping Rule for a Project with Uncertain Completion Time and Partial Salvageability. IEEE Transactions on Engineering Management, 44(1), 54-66. http://dx.doi.org/10.1109/17.552808

Deshmukh, S., \& Chikte, S. (1977). Dynamic investment strategies for a risky R and D project. Journal of Applied Probability, 14(1), 144-152.

Kamien, M., \& Schwartz, N. (1971). Expenditure patterns for risky R\&D projects. Journal of Applied Probability, $8(1), 60-73$.

Li, X., \& Liu, B. (2006). New Independence Definition of Fuzzy Random Variable and Random Fuzzy Variable. World Journal of Modelling and Simulation, 2(5), 338-342.

Liu, B. (2002). Theory and Practice of Uncertain Programming. Heidelberg: Phisica-Verlag.

Liu, B. (2004). Uncertainty Theory: An Introduction to Its Axiomatic Foundations. Berlin: Springer-Verlag.

Liu, B., \& Liu, Y. (2002). Expected Value of Fuzzy Variable and Fuzzy Expected Value Models. IEEE Transactions on Fuzzy Systems, 10(4), 445-450. http://dx.doi.org/10.1109/TFUZZ.2002.800692

Liu, Y., \& Liu, B. (2003). Expected Value Operator of Random Fuzzy Variable and Random Fuzzy Expected Value Models. International Journal of Uncertainty, Fuzziness and Knowledge-Based Systems, 11(2), 195-215.

Mehrez, A., \& David, I. (1999). A two-phase simple model to determine the timing and duration of R\&D project tasks. Production Planning $\mathcal{E}$ Control, 10(1), 48-53. http://dx.doi.org/10.1080/095372899233415

Nahmias, S. (1978). Fuzzy variables. Fuzzy Sets and Systems, 1(2), 97-110. http://dx.doi.org/10.1016/01650114(78)90011-8

Posner, M., \& Zuckerman, D. (1990). Optimal R\&D Programs in a Random Environment. Journal of Applied Probability, 27(2), 343-350.

Spall, J. (2003). Introduction to Stochastic Search and Optimization: Estimation, Simulation, and Control. New Jersey: John Wiley \& Sons.

Spector, Y., \& Zuckerman, D. (1997). Expenditure Patterns and Timing of Patent Protection in a Competitive R\&D Environment. Operations Research Letters, 20(5), 237-242. http://dx.doi.org/10.1016/S01676377(97)00009-6

Watanabe, C., Reshmin, S., \& Taras'yev, A. (2001). A dynamic model of R and D investment. Journal of Applied Mathematics and Mechanics, 65(3), 395-410. http://dx.doi.org/10.1016/S0021-8928(01)00045-4

Zhao, R., Tang, W., \& Yun, H. (2006). Random Fuzzy Renewal Process. European Journal of Operational Research, 169(1), 189-201. doi:10.1016/j.ejor.2004.04.049 
Zhao, G., \& Chen, W. (2009). Enhancing R\&D in science-based industry: An optimal stopping model for drug discovery. International Journal of Project Management, 27(8), 754-764. http://dx.doi.org/10.1016/j.ijproman.2009.01.003

Zuckerman, D. (1980). A diffusion process model for the optimal investment strategies of an R\&D project. Journal of Applied Probability, 17(3), 646-653.

\section{Copyrights}

Copyright for this article is retained by the author(s), with first publication rights granted to the journal.

This is an open-access article distributed under the terms and conditions of the Creative Commons Attribution license (http://creativecommons.org/licenses/by/3.0/). 